\title{
PROCESO DE CUIDADOS DE ENFERMERÍA. UN ABORDAJE EN URGENCIAS A UNA PACIENTE CON TRASTORNO DEPRESIVO
}

\section{THELMA FERNANDA RAMÍREZ-HERNÁNDEZ ${ }^{1}$ Y LUCÍA CAUDILLO-ORTEGA²}

${ }^{1}$ Estudiante de Licenciatura en Enfermería y Obstetricia. Departamento de Enfermería y Obstetricia. Universidad de Guanajuato (México).

2Doctora en Ciencias de Enfermería. Departamento de Enfermería y Obstetricia. Universidad de Guanajuato (México).

\section{INTRODUCCIÓN}

La depresión es considerada un problema de salud pública por su alta prevalencia y consecuencias adversas en la salud de la población ${ }^{1}$. La Organización Mundial de la Salud refiere que es el trastorno mental de mayor prevalencia y grado de discapacidad, que afecta a alrededor de 350 millones de personas en todo el mundo y se presenta mayoritariamente en mujeres ${ }^{2}$. Los pacientes con depresión pueden experimentar gran deterioro en su funcionamiento habitual, en su bienestar y también en su calidad de vida. La intervención efectiva en una crisis de depresión es un aspecto fundamental y vital para evitar los desenlaces mortales ${ }^{3}$.

Enfermería es el personal sanitario que se encuentra directamente relacionado con la atención de los pacientes. El proceso de atención de enfermería constituye una herramienta indispensable para el cuidado integral y continuo de los pacientes desde el área de urgencias.

Correspondencia: Lucía Caudillo-Ortega

Correo electrónico: lucia.caudillo@ugto.mx

\section{CASO CLÍNICO}

Mujer de 27 años de edad que ingresa en el área de urgencias del hospital psiquiátrico. La paciente luce desaliñada, tiende a permanecer en una misma posición durante mucho tiempo y tiene nula comunicación. Refiere sentirse cansada y con alteraciones del sueño. Manifiesta no tener apetito. Dice experimentar sentimientos de culpa por su enfermedad. Asimismo, presenta pérdida de interés por las cosas, dificultad para concentrarse y persistentes ideas suicidas, de una duración aproximada de tres semanas. Se trata de una segunda crisis de depresión en un período de ocho meses, con un intento de suicido previo. No ha recibido anteriormente atención profesional de salud. Es ingresada en la sala de observación y, posteriormente, a las seis horas, es trasladada a la sala de prehospitalización, donde permanece 72 horas antes de ser ingresada en el pabellón de mujeres para vigilancia intensiva por su alto riesgo de suicidio. Se inicia tratamiento con antidepresivos, estabilizadores del ánimo y antipsicóticos. 


\section{VALORACIÓN}

Para la recolección de datos, se realizaron tres técnicas: la observación, las entrevistas no estructurada y estructurada basada en los 13 dominios y la historia clínica (tabla 1). Posteriormente, se organizaron, se obtuvieron las etiquetas diagnósticas, se formularon los diagnósticos enfermeros según la taxonomía NANDA (North American Nursing Diagnosis Association $)^{4} \mathrm{y}$, a continuación, se establecieron los resultados y las intervenciones de enfermería con sus respectivas actividades, considerando como referentes las clasificaciones $\mathrm{NOC}^{5}$ y $\mathrm{NIC}^{6}$, respectiva- mente (tabla 2); y, finalmente, se realizó la evaluación.

En la priorización, se consideró la red de razonamiento de Daniel Pesut ${ }^{7}$. Esta se basa en la representación gráfica de la relación que existe entre los diferentes diagnósticos enfermeros (DE) encontrados a través de la valoración por dominios y el diagnóstico médico. Su propósito es conectar los DE con el problema principal. Se representa con flechas que van en dirección hacia el problema derivado. El problema principal es aquel al que apuntan un mayor número de flechas (fig. 1).

Tabla 1. Valoración por dominios

\begin{tabular}{|c|c|}
\hline Dominio & Valoración \\
\hline 1. Promoción de la salud & Se observa con descuido personal, en higiene, vestido y calzado. \\
\hline 2. Nutrición & Ingesta de alimentos disminuida en calidad y cantidad. \\
\hline 3. Eliminación & Diuresis y evacuaciones presentes. \\
\hline 4. Actividad-reposo & Períodos de insomnio e hipersomnia, catatonia. \\
\hline 5. Percepción-cognición & Mutismo. \\
\hline 6. Autopercepción & Sentimientos de culpa, baja autoestima e ideación suicida, anhedonia. \\
\hline 7. Rol-relaciones & Mala relación con su expareja. \\
\hline 8. Sexualidad & Sin alteraciones. \\
\hline 9. Afrontamiento-tolerancia al estrés & Pérdida de mecanismos. \\
\hline 10. Principios vitales & Culpabilidad por su hijo, se siente molesta con Dios. \\
\hline 11. Seguridad-protección & $\begin{array}{l}\text { La paciente tiene riesgo de autolesionarse por intentos suicidas } \\
\text { previos e ideas suicidas. }\end{array}$ \\
\hline 12. Confort & No refiere datos. \\
\hline 13. Crecimiento-desarrollo & No refiere datos. \\
\hline
\end{tabular}


Tabla 2. Diagnósticos NANDA, resultado NOC e intervenciones NIC

Diagnóstico (00150) Riesgo de suicidio r/c desesperanza

\begin{tabular}{|c|c|}
\hline NOC & $\begin{array}{l}1204 \text { Equilibrio emocional } \\
120416 \text { Ideas suicidas }\end{array}$ \\
\hline NIC & $\begin{array}{l}6340 \text { Prevención del suicidio } \\
\text { - Aumentar la observación y vigilancia. } \\
\text { - Retirar objetos que puedan utilizarse para autolesionarse. } \\
\text { - Utilizar intervenciones protectoras como restricciones de zonas. }\end{array}$ \\
\hline
\end{tabular}

Diagnóstico (00222) Control de impulsos ineficaz r/c pensamiento suicida y trastorno del estado del ánimo $\mathrm{m} / \mathrm{p}$ actuar sin pensar

\begin{tabular}{|c|c|}
\hline NOC & $\begin{array}{l}1408 \text { Autocontrol del impulso suicida } \\
140805 \text { Verbaliza control de impulsos }\end{array}$ \\
\hline NIC & $\begin{array}{l}5330 \text { Control del estado de ánimo } \\
\text { - Evaluar el estado de ánimo de la paciente. } \\
\text { - Ayudar a que la paciente hable de sus emociones, limitando el tiempo para las ideas } \\
\text { negativas. } \\
\text { - Ayudar a la paciente a expresar las ideas negativas de forma adecuada. } \\
\text { - Favorecer la expresión de sentimientos. } \\
\text { - Enseñar a la paciente estrategias de afrontamiento y entrenamiento del control de los actos } \\
\text { impulsivos. } \\
\text { - Reforzar la expresión de sentimientos. }\end{array}$ \\
\hline
\end{tabular}

Diagnóstico (00241) Deterioro de la regulación del estado de ánimo r/c desesperanza m/p aislamiento social, cambio en el apetito, cambios en el patrón de sueño, cambios en el peso
NOC
1409 Autocontrol de la depresión
140909 Refiere mejoría en el estado de ánimo
†PI: $1 ; \mathrm{PA}: 3$

\section{Control del estado de ánimo}
- Evaluar el estado de ánimo inicialmente y con regularidad, a medida que progresa el tratamiento.
- Administrar medicamentos estabilizadores del estado de ánimo (antidepresivos).
NIC - Vigilar y fomentar el cumplimiento de la medicación por parte de la paciente.
- Vigilar el estado físico de la paciente (peso e hidratación).
- Ayudar a la paciente a mantener un ciclo normal de sueño/vigilia (tiempos de reposo programados, técnicas de relajación).
- Animar a la paciente a que adopte un papel activo en el tratamiento.
- Darle la oportunidad de hablar acerca de sus sentimientos a través de un diario.

m/p: manifestado por; PA: puntuación alcanzada; PI: puntuación al ingreso; r/c: relacionado con.

* Escala desde 1 (siempre demostrado) hasta 5 (nunca demostrado).

† Escala desde 1 (nunca demostrado) hasta 5 (siempre demostrado). 
Figura 1. Red de razonamiento.

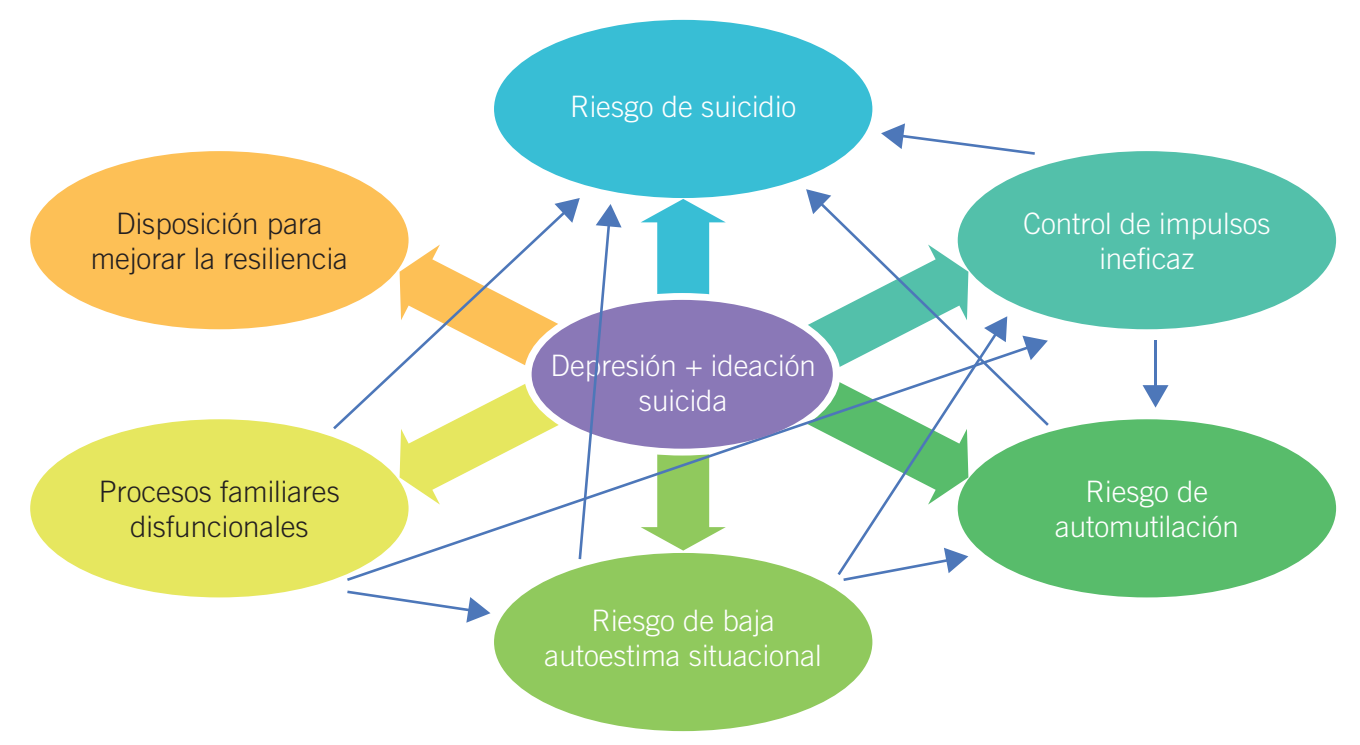

\section{EVALUACIÓN}

Inicialmente, se encontró a la paciente con alto riesgo suicida, estado de ánimo triste y mutismo. Tras las primeras $72 \mathrm{~h}$ de la prehospitalización, se observa una mejora de la apariencia física (baño e higiene dental), un ciclo de sueño con mejora en la frecuencia y duración, y control de la ideación suicida y la desesperanza a través de la medicación prescrita, la escucha activa y la vigilancia intensiva. Finalmente, se incorporó a la unidad de hospitalización de estancia corta para mujeres, así como a la psicoterapia familiar e individual con citas programadas dentro de la misma unidad.

\section{BIBLIOGRAFÍA}

1. Vincent GK, Velkoff V. The next four decades. The older population in the United States: 2010 to 2050. US Census Bureau. 2010;25:1138-48.
2. Organización Mundial de la Salud. Día Mundial de la Salud Mental: la depresión es el trastorno mental más frecuente [en línea]. 2012. Disponible en: http://www2. paho.org/hq/index.php?option=com_content\&view= article\&id=7305\%3A20

3. Navas W, Vargas MJ. Abordaje de la depresión: intervención en crisis. Revista Cúpula. 2012;26(2):19-35.

4. NANDA International. Diagnósticos enfermeros. Definiciones y clasificación 2015-2017. Barcelona: Elsevier; 2016.

5. Moorhead S, Johnson M, Maas ML, Swanson E (eds.). Clasificación de Resultados de Enfermería (NOC). Medición de Resultados en Salud. 5. ${ }^{a}$ ed. Barcelona: Elsevier; 2014.

6. Bulechek GM, Butcher HK, Dochterman JM, Wagner C (eds.). Clasificación de Intervenciones de Enfermería (NIC). 6. ${ }^{a}$ ed. Barcelona: Elsevier; 2014.

7. Pesut DJ. The art science and complexity of clinical reasoning. En: Oud N (ed.). Acendio 2007, 6th European conference of Acendio. Ámsterdam: Oud Consultancy; 2007. p. 33-7. 